\title{
Ingegnerizzazione dei fattori umani e sicurezza dei pazienti
}

\author{
Giulio Marcon ${ }^{1}$
}

Risk Manager Clinico. Professore di Management Sanitario e Management Clinico. Università di Padova

\begin{abstract}
Human Factors Engineering (HFE) is a discipline concerned with the analysis, design, and development of human-technological systems in which the primary emphasis is to improve or optimize system performance by considering the human's capabilities and limitations in the system. This article describes the applications of HFE to healthcare organization, to improve the performance of healthcare professionals and to reduce hazards.
\end{abstract}

Keywords: Human Factors Engineering, patient safety, performance, medical error

Human Factors Engineering and patient safety

Pratica Medica \& Aspetti Legali 2009; 3(1): 35-43

\section{PARADIGMI DELLA SICUREZZA}

L'obiettivo di migliorare la sicurezza dal paziente ha favorito la nascita di una serie di modelli interpretativi e operativi destinati a organizzare gli sforzi indirizzati al miglioramento della sicurezza stessa [1].

I modelli via via progettati o proposti per migliorare la sicurezza del paziente sono numerosi, ma possono essere riferiti a tre categorie di obiettivi:

- miglioramento dei risultati raggiunto con l'ottenimento di una riduzione degli errori dei professionisti;

- miglioramento dei risultati raggiunto mediante la riduzione dei danni ai pazienti;

- miglioramento e diffusione dell'applicazione della medicina basata sulle evidenze.

Il primo modello propone di migliorare la sicurezza del paziente mediante prevenzione, riduzione ed eliminazione degli errori nella fornitura delle cure. In quest'ottica si muove principalmente l'IOM (Institute of Medicine) [2]. In questo modello la riduzione degli errori può essere ottenuta es- senzialmente mediante la progettazione di sistemi sicuri.

Il secondo modello si basa sull'osservazione che errori e danni non sempre sono legati tra di loro e propone di occuparsi primariamente del danno [3]. Gli errori senza danno possono manifestarsi essenzialmente quando:

- l'errore avviene, ma non crea danno per situazioni fortuite (es. a un paziente viene somministrato il farmaco errato ma il paziente si dimentica di assumerlo);

- l'errore non è tale da creare un danno (es. la dose di farmaco assunta non è pericolosa);

- l'errore viene intercettato prima che causi un danno (ad es. quando qualcuno si accorge in tempo dell'errore e impedisce che si crei il danno).

Il terzo modello propone l'implementazione delle pratiche basate sulle evidenze. L'assunzione alla base di questo modello è che, se un medico segue le pratiche basate sulle evidenze, il paziente riceverà cure più accurate, in quanto le cure di qualità sono per definizione cure sicure [4].

Il paradigma che si focalizza sulla riduzione degli errori richiede che si manifesti un errore per far 
scattare un intervento mirato a migliorare la sicurezza del paziente, ed è più proattivo del secondo paradigma, che si focalizza solamente sui danni. Tuttavia entrambi i paradigmi possono essere visti come proattivi in quanto le cause che hanno determinato un outcome negativo importante (come ad esempio un errore o un danno) vengono comunque identificate, per cui è necessario impegnarsi in una riprogettazione organizzativa (ingegnerizzazione dei processi oltre all'ingegnerizzazione dei fattori umani) per prevenire danni in futuro.

Il terzo paradigma, ossia una più estesa utilizzazione della Evidence Based Medicine, offre un approccio totalmente diverso dagli altri due e in parte li completa.

Focalizzandosi sulla Evidence Based Medicine si può ridurre il danno al paziente ma, poiché gli errori e i danni continuano a manifestarsi, il terzo paradigma necessita degli altri due paradigmi per meglio garantire la sicurezza del paziente.

I tre modelli citati si basano sulla convinzione che, per ottenere la sicurezza del paziente, sono necessari cambiamenti radicali del sistema di fornitura delle cure. Esiste infatti la probabilità che, grazie ad una migliore progettazione dei sistemi di fornitura delle cure, gli errori e i danni possano essere eliminati, grazie anche all'implementazione della medicina basata sulle evidenze. Purtroppo non vi sono certezze sui meccanismi con i quali sia possibile ridurre gli errori e migliorare la qualità.

Tutti e tre questi modelli interpretativi, benché utili per migliorare la sicurezza del paziente, richiedono, per essere completi, di essere implementati attraverso un sistema che tenga conto dei comportamenti dei professionisti che forniscono le cure, per ottenere una performance migliore di quella attuale in modo da ottenere contemporaneamente una riduzione dei rischi di danno.

Il sistema che può essere utilizzato a tal fine è rappresentato dall'ingegnerizzazione dei fattori umani.

\section{L'INGEGNERIZZAZIONE DEI FATTORI UMANI (HFE)}

L'ingegnerizzazione dei fattori umani (Human Factors Engineering, HFE) è una disciplina che si occupa generalmente della progettazione di macchine, strumenti e sistemi con un'attenzione dedicata alle capacità umane, e alle limitazioni umane [1]. Gli obiettivi della HFE sono costituiti da messa in opera di progetti in grado di garantire la sicurezza del paziente con un alto grado di affidabilità.

Le tecniche usate vanno dall'ergonomia all'ingegnerizzazione delle condizioni d'uso e alla pro- gettazione centrata sull'utilizzatore: queste tre discipline sono considerate simili e molto vicine tra di loro.

Il processo della HFE è la base per i progetti eseguiti con la tecnica della "progettazione centrata sull'utilizzatore", la quale si focalizza sui bisogni dell'utilizzatore stesso, sulle sue caratteristiche, e sulla verifica finale dell'utilizzabilità interfaccia uomo-macchina. Un'altra caratteristica dell'approccio basato sull'utilizzatore è costituita dal concetto della progettazione iterativa e dal controllo iterativo dei risultati. Il processo progettuale viene continuamente e ripetutamente esaminato e migliorato mediante cicli iterativi di progettazione, mediante l'analisi del feedback da parte dell'utilizzatore (valutazione dell'utilizzabilità), dall'applicazione del progetto e dalla verifica dei risultati. In questo modo è più facile ottenere un monitoraggio continuo dell'andamento del progetto e del raggiungimento dei risultati voluti.

Con questo metodo inoltre, e in particolare con la valutazione e la verifica continua dell'utilizzabilità, i difetti di progettazione possono essere corretti e rettificati prima della messa in atto definitiva del progetto grazie all'uso dei test di controllo ripetuti.

Una semplice riprogettazione del sistema non comporta automaticamente il miglioramento della qualità delle cure. La riprogettazione del sistema influisce sui risultati mediante il miglioramento dei processi di cura, miglioramento che dipende da due elementi specifici:

- miglioramento della performance. La performance in campo sanitario può essere definita come la serie di attività correlate con la cura dei pazienti (fisiche, mentali, sociali, e comportamentali) portate a termine dai professionisti per ottenere un risultato positivo in termini di salute.

- riduzione dei rischi. I rischi sono un elemento di sistema che aumenta la probabilità di un errore o di un danno.

Il modello dell'ingegnerizzazione dei fattori umani delle cure si propone di migliorare la sicurezza dei pazienti influendo su entrambi questi elementi: secondo questo paradigma, quindi, la progettazione organizzativa deve essere in grado di supportare la performance dei professionisti e di eliminare, o almeno ridurre, i rischi.

La HFE è una scienza che si applica alla definizione dei sistemi che valuta le forze dell'uomo e ne compensa i limiti. Di conseguenza, conoscere il modo in cui gli individui processano e interpretano l'informazione è essenziale per sviluppare un sistema di questo tipo. Gli individui ricevono degli imput esterni che, attraverso un processo di interpretazione, vengono "trasformati" per dar vita a degli output, ossia a delle azioni o decisioni specifiche. È all'interno di questo processo che possono verificarsi gli errori: comprenderne le metodiche e 
le peculiarità, quindi, è fondamentale per capire quale sia la fonte dell'errore, per intervenire sul processo al fine di migliorare la performance del soggetto e, in definitiva, incrementare la qualità dell'intero sistema di cure.

Ad esempio estrarre le informazioni dall'ambiente esterno può essere condizionato da fattori che dipendono dall'ambiente stesso: lavorare in un ambiente rumoroso e caotico, per esempio, determina un accesso limitato alle informazioni, che possono essere quindi mal interpretate o raccolte in modo insufficiente. Inoltre, l'errore nell'interpretare l'input può derivare da informazioni discordanti. Un esempio può essere rappresentato da un farmaco in soluzione che è stato studiato per una somministrazione orale ma che è stato erroneamente inserito in una siringa. In questo caso la persona addetta alla somministrazione può ignorare la nozione, di cui magari è a conoscenza, sulla corretta via di somministrazione prevista per quel dato prodotto, poiché è tratta in inganno dalla presentazione in siringa (uso parenterale). Un esempio di questo tipo mostra come spesso gli individui siano portati a rispondere in modo automatico a ciò che percepiscono come familiare.

\section{LA PERFORMANCE}

La performance dei professionisti può essere definita come "il modello delle azioni eseguite allo scopo di raggiungere un obiettivo soddisfacendo degli standard definiti».

Questa e altre definizioni della performance si basano sulle azioni, sulle attività e in generale sui "processi di trasformazione" che i professionisti mettono in atto per ottenere un risultato.

Le trasformazioni costituiscono uno stadio susseguente di quello che viene chiamato un input (letteralmente "ingresso") che può essere definito come una precondizione del sistema che ha influenza sulla performance. In termini generali un input può essere costituito da una modifica (trasformazione) dello stato del paziente che crea una trasformazione nel paziente stesso. Le interazioni fra i vari input (ad es. un'attività interprofessionale) contribuiscono a garantire la qualità delle trasformazioni, le quali a loro volta generano i risultati (output), secondo un processo che può essere schematizzato come input $\rightarrow$ trasformazione $\rightarrow$ output.

Anche se input, gli output e le trasformazioni vengono intesi come unità separate, essi in realtà rappresentano i vari aspetti della fornitura delle cure, e soprattutto costituiscono le tappe delle attività cognitive che il nostro cervello esegue continuamente.

\section{GLI INPUT}

Gli input del sistema nel quale i professionisti esercitano il loro lavoro costituiscono contemporaneamente anche input per la performance.

Questi input includono (Tabella I):

- fattori del paziente e del professionista: stato di salute, età, peso, bisogni, umori. personalità, esperienza, intelligenza, curiosità, comunicazione, linguaggio, conoscenze;

- fattori del compito singolo o dell'intera procedura: conoscenza dell'attività, del processo o della procedura che il professionista deve eseguire, il che comprende la conoscenza e l'esecuzione puntuale delle modalità di esecuzione delle attività, del processo o della procedura, la quantità di tempo necessaria per eseguire le attività, il controllo dei risultati, il carico totale di lavoro;

- fattori legati all'uso di tecnologie: i fattori relativi all'uso di tecnologie necessarie per il lavoro dei professionisti sanitari sono riferibili al numero, alla complessità, alla qualità, alla quantità, all'affidabilità e al tipo di tecnologie presenti nell'organizzazione. Altri fattori legato all'uso delle tecnologie sono costituiti dalla loro disponibilità, dalla loro numerosità, dalla loro collocazione, dalla loro trasportabilità, dalla loro possibilità di integrazione con tecnologie diverse, dalla loro facilità di uso, dalla loro affidabilità e dalla loro robustezza;

- fattori ambientali interni: sono costituiti dall'ambiente fisico, dall'illuminazione, dal rumore, dalle condizioni microclimatiche, dallo spazio disponibile, dalla disposizione generale delle aree di lavoro, dai percorsi;

- fattori ambientali esterni: l'ambiente esterno di un'organizzazione è rappresentato da tutto ciò in cui è immerso l'ambiente interno. Le "dimensioni" dell'ambiente interno e di quello esterno possono avere metri di misura molto differenti: l' "interno" di un paziente ricoverato può essere la stanza di degenza, mente l' "esterno" di un paziente ricoverato in una stanza è costituito dal corridoio ("esterno immediato"), ma può comprendere, in un'accezione estesa, anche le leggi statali o locali, il contesto abitativo, il contesto lavorativo, le condizioni economiche e la demografia di un determinato ambito geografico ("esterno esteso");

- fattori organizzativi: politiche strutturali e culturali correlate con le caratteristiche dell' organizzazione. Ne sono esempi la leadership, il management, la cultura, le regole, i livelli di gerarchia, i livelli e i tipi di supervisione e di controllo.

Gli input citati non rappresentano una serie esaustiva di tutti gli input da considerare e nessuno 
degli input citati è di per sé in grado di mettere in forse la sicurezza dal paziente. Tuttavia, se più input interagiscono tra di loro, è possibile che la sicurezza del paziente sia a rischio. Si può paragonare l'effetto degli input all'illuminazione: l'illuminazione in genere non è né buona né cattiva, ma l'illuminazione insufficiente può essere causa di problemi (come ad esempio l'eccesso di illuminazione o la creazione di bagliori in alcuni display).

Anche una tecnologia raffinata (come ad esempio il sistema computerizzato di prescrizione dei farmaci) può influenzare in alcuni casi la sicurezza del paziente se usata in modo scorretto. Il che significa che in alcune condizioni operative la prescrizione computerizzata può causare effetti negativi, in altre effetti positivi. La possibilità che gli effetti positivi o negativi si manifestino realmente dipende da input che possono mettere in forse la qualità della prescrizione (il che può avvenire nel caso in cui la prescrizione computerizzata non sia interfacciata con la cartella elettronica del paziente) e quindi mettere in forse la sicurezza del paziente. Questi input, ad esempio nel modello della prescrizione computerizzata, possono essere:

- il livello medio di integrazione della prescrizione computerizzata con la cartella elettronica nel contesto di lavoro;

- il livello di addestramento raggiunto dai professionisti che usano questo tipo di prescrizione;

- la percezione degli utilizzatori relativa alla facilità d'uso della prescrizione computerizza- ta nel contesto di lavoro e alla sua reale utilizzabilità.

Va ricordato che ogni sistema locale (come ad esempio un ospedale, una corsia ospedaliera o un ambulatorio) da un lato alberga dentro di sé alcuni sistemi di ordine inferiore i quali contengono a loro volta altri sottosistemi (o microsistemi) e dall'altro costituisce parte di un sistema di maggiori dimensioni (come ad esempio una rete di ospedali). Ogni livello del sistema generale si compone infatti di una fitta rete di microsistemi. Nel caso di un'unità operativa ospedaliera il sistema/ unità operativa comprende le regole organizzative e operative, lo staff infermieristico, lo staff medico, lo staff di supporto, i luoghi di lavoro dedicati, le procedure formalizzate (come ad esempio la somministrazione dei farmaci). Ogni parte del sistema (ad esempio lo staff infermieristico di una corsia ospedaliera) da un lato copre un'area definita delle cure e dall'altro si articola con i sistemi di ordine superiore o con i sistemi di ordine inferiore (come ad esempio un ambulatorio o un gruppo di lavoro dedicato). Le gerarchie tra i vari livelli del sistema sono importanti in quanto influenzano il lavoro dei professionisti, la loro performance e la sicurezza dal paziente [5].

Tutto ciò implica che la progettazione o la riprogettazione di una qualunque parte di un'organizzazione sanitaria deve prendere in esame con attenzione:

- il contesto in cui la parte dell'organizzazione opera (il che richiede l'analisi e la valutazione dei sistemi di ordine superiore da un lato

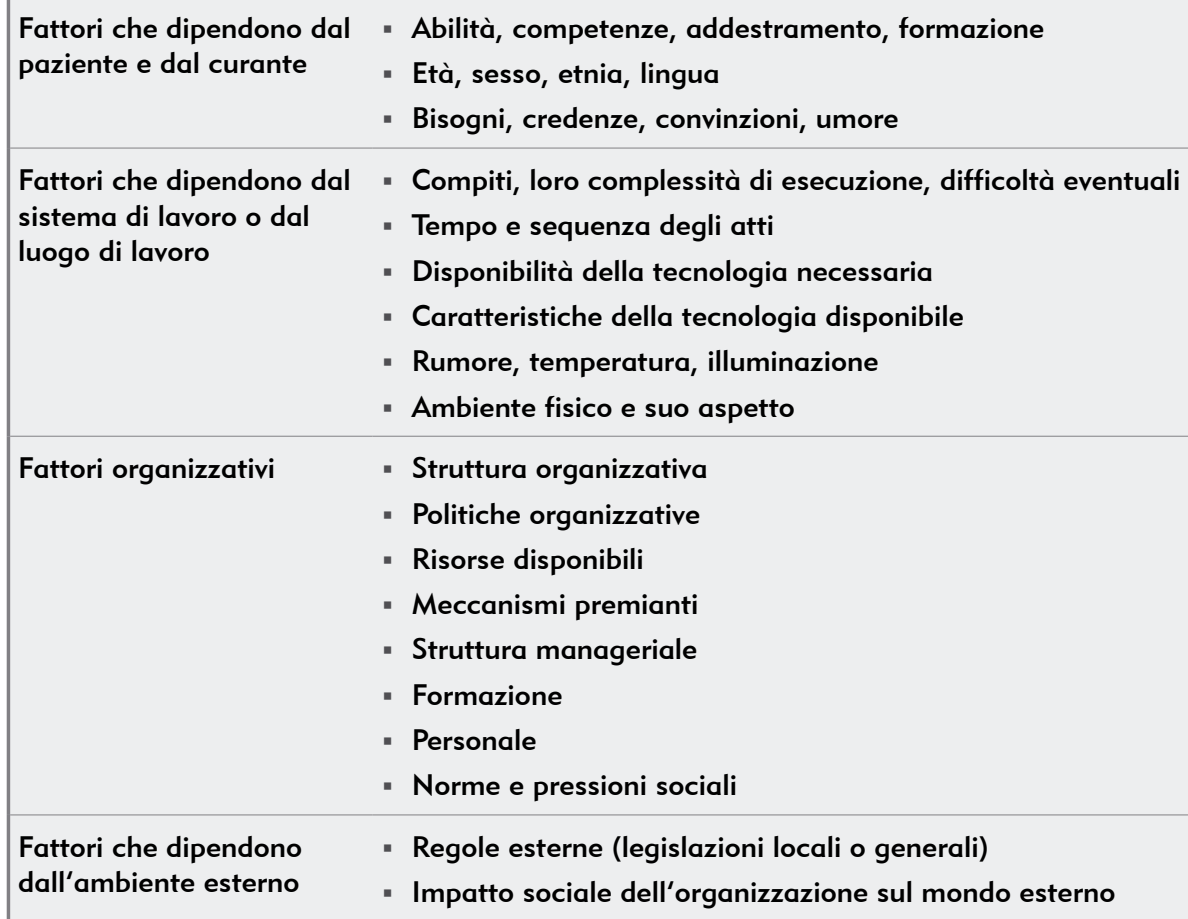

Tabella I. Input relativi alla performance 
e l'impatto sui microsistemi di ordine inferiore dall'altro).

- l'impatto prevedibile degli input e degli output tra i vari livelli di sistema.

\section{LE TRASFORMAZIONI}

I passaggi del paziente tra i vari livelli del sistema sono scanditi dalle trasformazioni. Le trasformazioni sono modifiche dello stato fisico causate dagli input che generano gli effetti dei processi sanitari e garantiscono la performance dei processi stessi sul paziente. Il processo di trasformazione si compone di input (elementi in entrata come storia clinica, sintomi, patologie, decisioni cliniche) che creano degli output (risultati desiderati delle cure).

I processi di trasformazione possono essere evidenti, ad esempio un paziente che viene trasferito a un'altra unità per ottenere altre cure oppure un paziente che è stato operato con successo (ossia ha subito fisicamente una trasformazione). Oltre queste trasformazioni "visibili" esistono anche trasformazioni che operano sul piano cognitivo/sociale, come avviene ad esempio quando un medico esegue una diagnosi difficile (input), dopo la quale il medico è mentalmente affaticato e il paziente cognitivamente più informato di quanto non lo fosse prima (output).

Il paziente è naturalmente coinvolto in tutte le fasi del processo di trasformazione.

Nella fase di input del processo le caratteristiche del paziente determinano in parte il comportamento del sistema, il quale deve adattarsi alla situazione del paziente. Ad esempio, possono essere necessari letti e strumenti diversi per bambini e per adulti o carrozzine particolari per disabili. Le trasformazioni costituiscono anch'esse attività correlate al paziente, come ad esempio nel caso in cui il malato viene coinvolto nelle decisioni terapeutiche che lo riguardano. L'output genera un cambiamento nel paziente e quindi nel sistema, e si riferisce ai cambiamenti per il malato, il quale riceve una cura che genera una trasformazione, come ad esempio la guarigione di una malattia.

Nella progettazione del sistema è necessario tenere in conto tre tipi di performance: fisica, cognitiva e socio/comportamentale.

\section{GLI OUTPUT}

Un output di performance può essere costituito dall'esecuzione di un processo di trasformazione (ad es. "intervento chirurgico terminato" oppure "somministrato il farmaco al paziente"). L'output può anche essere inteso come uno stato di pretrasformazione, che deve essere completato e verificato per garantire la sua adesione agli standard predefiniti (nella somministrazione dei farmaci, ad esempio, è necessario somministrare il farmaco giusto, al paziente giusto, all'ora giusta, nella dose giusta, per la via giusta). Per la sicurezza del paziente è necessario non solo che vengano eseguite alla lettera le regole, ma anche che le informazioni vengano date correttamente alla persona giusta.

Se gli output raggiungono gli obiettivi e rispettano gli standard di sicurezza, qualità ed efficien$\mathrm{za}$, agiscono anche come input per altri processi successivi favorendone la corretta esecuzione nel tempo.

La somministrazione dei farmaci può, ad esempio, essere influenzata da vari input. L'output della somministrazione è costituito dalla correttezza o meno della somministrazione. Se dai dati di somministrazione si nota che si verificano errori nella sequenza degli atti (input $\rightarrow$ trasformazione $\rightarrow$ output), questa conoscenza permette di identificare in termini di feedback correttivo i problemi nella fase di somministrazione e permette di pianificare una rivalutazione del processo di trasformazione e di adottare le misure correttive necessarie (uso di tecnologie specifiche, modifiche organizzative, nuove politiche) in modo da garantire che i professionisti abbiano una performance migliore e garantiscano quindi una migliore sicurezza del paziente.

\section{TIPOLOGIE DI PERFORMANCE}

\section{Performance fisica}

La performance fisica si riferisce alle attività fisiche eseguite mediante il sistema muscoloscheletrico. Camminare, spingere un carrello, palpare, trasferire pazienti, costituiscono altrettanti esempi di performance fisiche. Come in tutti gli altri tipi di performance, l'esecuzione corretta di una

\begin{tabular}{|c|c|}
\hline $\begin{array}{l}\text { Output } \\
\text { immediati }\end{array}$ & $\begin{array}{l}\text { - Cambiamenti dello stato fisico (curante, paziente) } \\
\text { - Cambiamento dello stato mentale (fatica, stress, } \\
\text { umore, stato di coscienza) (curante, paziente) } \\
\text { - Altri cambiamenti } \\
\text { - Comportamenti della famiglia } \\
\text { - Altri soggetti } \\
\text { - Compiti } \\
\text {. Tecnologia } \\
\text { - Organizzazione } \\
\text { - Ambiente fisico } \\
\text { - Ambiente sociale }\end{array}$ \\
\hline $\begin{array}{l}\text { Output } \\
\text { finali }\end{array}$ & $\begin{array}{l}\text { - Sicurezza del paziente } \\
\text { - Sicurezza dei dipendenti } \\
\text { - Qualità delle cure }\end{array}$ \\
\hline
\end{tabular}

Tabella II. Gli output della performance 
performance fisica dipende sia dall'individuo che sta eseguendo l'attività, che dal sistema nel quale l'attività viene eseguita. Alcuni input importanti per la performance fisica possono essere:

- caratteristiche individuali del professionista: forza, acuità visiva, abilità, flessibilità, età, esperienza,competenza, stanchezza, salute, forma fisica;

- caratteristiche del paziente: mobilità, peso, cooperazione, abilità comunicativa, coordinazione;

- caratteristiche del compito: numero di pazienti da visitare, numeri di farmaci da somministrare, flussi di lavoro;

- caratteristiche dipendenti dalla tecnologia e dalla strumentazione: disponibilità degli strumenti necessari, facilità di uso, qualità dei monitor;

- caratteristiche ambientali: disposizione dello spazio fisico, illuminazione, temperatura;

- caratteristiche organizzative: enfasi sulla velocità, sulla qualità, sulla sicurezza, politiche di finire i lavoro iniziati, norme sociali;

- caratteristiche dell'ambiente esterno: regolazioni relative all'orario di lavoro, convenzioni esterne per la fornitura di arredi e spazi di lavoro.

Per capire appieno l'influenza degli input sulle trasformazioni, è importante valutare l'effetto del sistema sulla performance. Ad esempio, la forza e la stanchezza di un'infermiera, il peso e la mobilità di un paziente, l'impegno continuo del personale, la disponibilità di trasporti per i pazienti, la disposizione fisica dell'unità, la pressione "sociale" derivante dall'organizzazione, messe tutte assieme determinano un'interruzione dei processi, come potrebbe essere ad esempio la richiesta di un'infermiera a farsi aiutare da una collega per sollevare un paziente particolarmente pesante.

Dato che gli output del processo di trasformazione della situazione fisica dei pazienti determinano una modifica del sistema, si possono considerare gli output stessi come prodotti finali (quando il processo di trasformazione ha ottenuto i suoi obiettivi) ma si possono anche definire come input se sono necessari altri e ulteriori cicli di trasformazione. Ad esempio, il farmacista considera i farmaci come output del processo di preparazione e distribuzione degli stessi, mentre l'infermiera considera i farmaci come un input necessario per avviare il processo di somministrazione dei farmaci stessi.

Una buona gestione dei fattori umani del lavoro può generare input di sistema che facilitano il lavoro e la performance dei professionisti che a loro volta generano output che vanno a favore del paziente (meno danni ed errori), dell'amministrazione (uso efficiente delle risorse) e dei professionisti (riduzione della fatica).

\section{Performance cognitiva}

La performance cognitiva è associata all'elaborazione delle informazioni ricevute, la quale genera trasformazioni nel cervello umano, comprese alcune funzioni del cervello stesso come percezione, pensiero, ragionamento, e ricordo.

Per comprendere come i differenti sistemi di lavoro possano interferire con la performance cognitiva è utile considerare le caratteristiche e gli input che provengono dagli elementi del sistema di lavoro che sono:

- caratteristiche individuali del professionista: conoscenze, memoria e capacità di attenzione;

- caratteristiche del paziente: lingua, educazione, background economico, salute mentale;

- caratteristiche del compito: complessità del compito, pressione del tempo, modalità di raccolta delle informazioni, interruzioni;

- caratteristiche dipendenti dalla tecnologia e dalla strumentazione: facilità d'uso, leggibilità, standardizzazione dei display, chiarezza delle informazioni;

- caratteristiche ambientali: illuminazione, uso degli spazi, rumore, distrazioni;

- caratteristiche organizzative: formazione, addestramento, attese del management, riconoscimento del lavoro fatto velocemente invece che del lavoro fatto bene;

- caratteristiche dell'ambiente esterno: politiche, relazioni, standard informatici relativi ai display che forniscono informazioni, tradizioni accademiche nella formazione dei professionisti.

Gli elementi umani e gli input dal sistema di lavoro possono interagire con la performance personale. Ad esempio, l'illuminazione, la pressione a lavorare in fretta, la complessità dei compiti, l'acuità visiva interagiscono tutti nel creare uno stato di tensione a causa del quale si può sbagliare la somministrazione di un farmaco.

Le trasformazioni cognitive sono importanti per il lavoro e sono comprensibili se si pensa che l'improvviso scattare di un allarme genera nell'individuo varie reazioni. La prima reazione è costituita dalla sensazione di un'anomalia e dalla ricerca della fonte del segnale, seguita dalla percezione vera e propria dell'allarme e dalla localizzazione della fonte. Il soggetto a questo punto accede alla sua memoria remota per determinare il significato dell'allarme. Poi l'individuo si impegna nel decision making nella ricerca di una risposta al perché è scattato un allarme e tenta di prendere una decisione sul da farsi. Successivamente l'individuo accede alla memoria di lavoro per mantenere attiva la risposta trovata $\mathrm{e}$ per decidere se finire l'attività interrotta dall'allarme, se sia utile spegnere l'allarme o se sia più utile 
rispondere all'allarme. Infine, l'individuo si impegna in un monitoraggio post-risposta per calibrare l'impatto del suo comportamento sul sistema.

Le trasformazioni cognitive possono produrre effetti diversi. In alcuni soggetti questi aspetti sono identificabili nei comportamenti verbali e motori, mentre in altri soggetti vengono influenzate attività come lo studiare, il sovraccarico mentale, il raggiungimento di decisioni.

ll progettare gli input in modo da supportare la performance cognitiva (ad esempio, riducendo le distrazioni e la dipendenza dalla sola memoria) può supportare le abilità dei professionisti e migliorare il rapporto segnale/rumore producendo output positivi (miglioramento della memoria e della comunicazione, decisioni corrette, fornire risposte accurate).

\section{Performance sociale/comportamentale}

La performance sociale/comportamentale si riferisce alle trasformazioni che non sono trattate dalla scienza cognitiva ma dalle scienze sociali. Queste trasformazioni includono i processi mentali usati per attribuire, ad esempio, una catena causale, oppure una forma di autoregolazione, oppure un comportamento socialmente accettabile.

Come negli altri tipi di performance una varietà di input di sistema può essere rilevante per la performance sociale/comportamentale:

- caratteristiche individuali del professionista: esperienza, età, abilità e/o limitazioni fisiche, conoscenze, personalità, situazione finanziaria, problemi familiari;

- caratteristiche del compito: pressione del tempo, complessità, eccessivo numero di compiti, flussi di lavoro, socializzazione;

- caratteristiche dipendenti dalla tecnologia e dalla strumentazione: grado di automazione, approccio alla tecnologia, necessità di assistenza del team nell'usare le tecnologie e gli strumenti;

- caratteristiche ambientali: tipo di spazi di lavoro, disponibilità di spazio;

- caratteristiche dell'organizzazione: sistemi premianti, stile manageriale, formazione, cultura;

- ambiente esterno: locale, regionale, statale aspetti particolari della cultura.

Gli input individuali e gli input derivanti dall'ambiente di lavoro interagiscono nell'influenzare le trasformazioni comportamentali e, di conseguenza, interagiscono con gli output.

Queste trasformazioni possono avere influenza su vari comportamenti come l'atteggiamento, le motivazioni, i piani e le decisioni.

Oltre a ciò, sono caratteristici nel mondo sanitario i comportamenti di reazione agli incidenti come la "ricerca del colpevole", le convinzioni dei singoli, le attitudini particolari, le decisioni relative all'uso di alcune tecnologie (modalità di utilizzo, omissione di passi) e i comportamenti deliberati (aggiramento di regole, mancato rispetto di protocolli importati come quello del lavaggio delle mani).

\section{Considerare insieme tutti i tipi di performance}

Come risulta evidente, gli elementi di un sistema e i differenti tipi di performance sono strettamente interconnessi tra di loro. Questo implica che nes-

\begin{tabular}{|c|c|}
\hline $\begin{array}{c}\text { Tipologia } \\
\text { di performance }\end{array}$ & $\begin{array}{l}\text { Azioni } \\
\text { correlate }\end{array}$ \\
\hline Fisica & $\begin{array}{l}\text { - Abbassare } \\
\text { - Adattare } \\
\text { - Alzarsi } \\
\text { - Camminare } \\
\text { - Correre } \\
\text { - Iniettare } \\
\text { - Manipolare } \\
\text { - Preparare } \\
\text { - Scrivere a mano } \\
\text { - Scrivere al computer } \\
\text { - Sedersi } \\
\text { - Trasportare } \\
\text { - Trovare }\end{array}$ \\
\hline Cognitiva & $\begin{array}{l}\text { - Analizzare } \\
\text { - Cercare } \\
\text { - Comunicare } \\
\text { - Controllare } \\
\text { - Immaginare } \\
\text { - Imparare } \\
\text { - Localizzare } \\
\text { - Percepire } \\
\text { - Porre attenzione in modo conscio } \\
\text { - Prevedere } \\
\text { - Risolvere problemi (problem solving) } \\
\text { - Riconoscere le configurazioni } \\
\text { - Vigilare }\end{array}$ \\
\hline Cognitivo/sociale & $\begin{array}{l}\text { - Attribuire un nesso di causalità } \\
\text { - Autoregolarsi } \\
\text { - Cercare una motivazione } \\
\text { - Effettuare un'analisi costi/benefici } \\
\text { - Pianificare il comportamento adeguato } \\
\text { - Prendere decisioni } \\
\text { - Ricordare } \\
\text { - Risolvere i problemi } \\
\text { - Vigilare }\end{array}$ \\
\hline
\end{tabular}

Tabella III. Tipologie di performance e relative azioni (trasformazioni) 
sun input può essere rilevante solo per un singolo processo di trasformazione, in quanto anche le piccole modifiche di un processo di trasformazione singola possono divenire nel tempo introiettate da professionisti, possono diventare cattive abitudini, che possono causare effetti rilevanti anche a distanza nel susseguirsi di cicli ripetuti di trasformazione.

Ad esempio, gli allarmi nelle corsie o in sala operatoria possono essere una fonte di distrazione per $\mathrm{i}$ professionisti, ma possono avere effetto anche su tutti i tipi di performance. Un suono inatteso può causare sia un disturbo di natura uditiva (interruzione della performance fisica), sia un'interruzione della comunicazione interpersonale (interruzione della performance sociale/comportamentale) sia un'interruzione dell'attenzione (alterazioni della performance cognitiva).

Gli input di sistema possono interferire con diversi tipi di performance sia in modo diretto che in modo indiretto anche quando gli effetti sembrano apparentemente poco importanti.

Tutto ciò implica che, quando bisogna pensare come progettare o riprogettare un sistema di cura, è necessario che il progetto sia coerente con ciascuno dei differenti tipi di performance che i professionisti devono imparare ed introiettare i differenti tipi di performance che differenti professionisti deve essere in grado di garantire.

\section{RISCHI}

Una volta che è stato compreso in che modo gli input di sistema influenzino la performance dei professionisti, si capisce come input mal progettati possano agire come rischi in quanto possono favorire una performance inadeguata aumentando quindi il pericolo di eventi avversi, errori, danni e scarsa qualità.

Si considera come un "pericolo" una situazione che aumenta la probabilità di errori e danni ai pazienti o ai dipendenti. Il "pericolo" corrisponde al termine "fattore di rischio" nelle cure mediche o nelle statistiche epidemiologiche.

I "pericoli" si manifestano frequentemente nel mondo del lavoro e tendono a interagire tra loro causando altri pericoli. Un esempio può essere rappresentato dall'utilizzo dei bracciali identificativi con i codice a barre, molto usati negli Stati Uniti. Tale sistema di identificazione del paziente è stato sviluppato per evitare gli errori. Tuttavia può essere anche fonte di rischi: ad esempio, quando un'infermiera usa uno scanner wireless per identificare un paziente dal suo braccialetto per poter somministrare i farmaci prescritti, la lettura delle informazioni sul display può essere resa difficoltosa da uno scarso contrasto tra il testo del messaggio e lo sfondo illuminato del display. La situazione di scarsa leggibilità del display può essere peggiorata in situazioni nelle quali la rifrazione della luce ambiente crea difficoltà alla lettura del testo per cui l'infermiera deve leggere il display con un innaturale piegamento del polso.

Le interazioni tra input diversi possono quindi comportare pericoli in quanto sono in grado di peggiorare molti tipi di performance, come la percezione visiva, la presa di decisioni, l'attività motoria, l'attenzione.

\section{CONCLUSIONI}

Il paradigma dell'ingegnerizzazione dei fattori umani per la sicurezza del paziente è utile come paradigma alternativo e complementare alla riduzione degli errori e dei danni, alla riduzione dei rischi e al paradigma basato sulle Evidence Based Medicine.

Questo modello si concentra principalmente sulla performance dei professionisti e sulla riduzione dei rischi, con lo scopo di ridurre gli errori e di innescare un ciclo di miglioramento della qualità.

Il punto di forza del modello dell'HFE, rispetto agli altri, è quello di rivolgersi ai professionisti spingendoli a usare processi di cura in grado di garantire i migliori risultati per i pazienti e per l'organizzazione. Una situazione particolare del paradigma dell'engineering dei fattori umani è costituita dalla necessità di progettare le azioni sia in base ai bisogni del paziente che in base alle decisioni del professionista. Tradizionalmente infatti ci si occupa per prima cosa dei problemi del paziente per migliorare la sua situazione di salute. Il paziente è parte del sistema ed è una fonte di input per il professionista che lo cura. Ma il paziente può anche costituire una fonte di pericolo, come ad esempio quando si autosomministra un farmaco senza avvertire i professionisti. Ne discende che il paziente può involontariamente modificare i risultati delle cure e creare problemi in termini di qualità e sicurezza. Inoltre l'HFE si focalizza sugli aspetti positivi del cambiamento: al contrario di un sistema basato solo sul tentativo di ridurre gli errori, che può essere fonte di frustrazione per il professionista (poiché finisce per sentirsi il colpevole unico dei danni dei pazienti), l'ingegnerizzazione dei fattori umani spinge ad adottare un punto di vista positivo, aiutando i professionisti a identificare i loro bisogni per migliorare le cure.

Infine tale modello non è slegato dagli altri, ma anzi ne è un complemento e un'implementazione. Alphonse Chapanis, uno dei fondatori dell'ergonomica, condusse nei primi anni '60 quello che può 
essere considerato uno dei primi studi sugli errori medici, identificando circa 250 tipologie di errore e le relative cause [6]. Ad oggi, molte delle raccomandazioni derivanti dallo studio di Chapanis non sono ancora entrate a far parte della mentalità e delle procedure che caratterizzano il lavoro delle organizzazioni sanitarie. È ovvio che la sola applicazione di modelli e paradigmi non può essere sufficiente a eliminare errori, violazioni e danni ai pazienti. Tuttavia, proprio a causa della complessità e delle criticità della realtà sanitaria, è fon- damentale sviluppare sistemi che aiutino a comprenderne i processi.

Lingegnerizzazione dei fattori umani può essere di notevole utilità in tal senso, consentendo di esplicitare le relazioni tra il contesto in cui si svolge il lavoro, le relative variabili e la sicurezza del paziente. Sarebbe quindi utile sviluppare delle ricerche specifiche in questo campo, che vedano protagonisti i professionisti sanitari e le organizzazioni attraverso una stretta collaborazione [7].

\section{APPLICAZIONI DELLA HFE NELL'IMPLEMENTARE LA SICUREZZA CORRELATA ALL’USO DEI FARMACI [8]}

- Vi deve essere coerenza tra l'aspetto di un oggetto e la sua funzione: ad esempio le formulazioni liquide che vanno usate per la somministrazione orale non vanno presentate in contenitori simili a siringhe, che posso indurre in errore causando una somministrazione parenterale

- Le informazioni che vengono fornite agli operatori nel momento in cui viene loro affidato un compito devono essere chiare, concise e tempestive: ad esempio le avvertenze per evitare il sovradosaggio devono essere incluse nel sistema usato per la prescrizione, sia esso un sistema manuale che computerizzato

- È necessario rendere minimo il numero di modi di fare una cosa e gli interventi devono essere univoci: ad esempio i device per il controllo elettronico dell'infusione non devono essere muniti di un solo meccanismo di controllo che attiva più di una funzione critica (es. programmare la quota di infusione e iniziare l'infusione)

- Le etichette devono essere chiare e senza ambiguità: per esempio i farmaci non devono essere immagazzinati in modo che le etichette risultino difficilmente leggibili, o in modo che possano essere facilmente confuse con farmaci differenti

- Devono essere disponibili risorse adeguate e procedure sufficienti a rimediare agli errori: ad esempio gli antidoti per i farmaci a elevate tossicità devono essere disponibili in modo rapido

- Il sistema deve essere strutturato in modo tale da fare il minor affidamento possibile sulla memoria: ai professionisti che utilizzano i farmaci devono essere resi disponibili e facilmente accessibili strumenti che forniscano tutti i dati relativi al farmaco in modo da supportarne le decisioni e chiarire immediatamente gli eventuali dubbi

- L'ambiente in cui avviene la somministrazione del farmaco deve essere adeguato, ad esempio per condizioni di illuminazione

\section{BIBLIOGRAFIA}

1. Karsh BT, Holden RJ, Alper SJ, Or CK. A human factors engineering paradigm for patient safety: designing to support the performance of the healthcare professional. Qual Saf Health Care 2006; 15: 59-65

2. Institute of Medicine. To Err is Human: building a safer healthcare system. Washington, DC: National Academy Press, 2000

3. Layde PM, Cortes LM, Teret SP, Brasel KJ, Kuhn EM, Mercy JA et al. Patient safety efforts should focus on medical injuries. JAMA 2002; 287: 1933-7

4. Brennan TA, Gawande A, Thomas E. Accidental deaths, saved lives and improved quality. N Engl J Med 2005; 353: 1405-9

5. Karsh B, Brown R. The inpact of organizational hierarchies on the design and analysis of medical error research. Proceedings of Human Factors in Organizational Design and Management 2005; 8: 293-8

6. Chapanis A, Safrin MA. Of misses and medicines. Journal of Chronic Diseases 1960;12: 403-8

7. Carayon $\mathrm{P}$ (a cura di). Handbook of human factors and ergonomics in health care and patient safety. New York: Routledge, 2006

8. Schneider PJ. Applying human factors in improving medication-use safety. Am J Health-Syst Pharm 2002; 59: 1155-9 